\title{
Stationary Wavelet Transform for the Extraction of the Impedance Circulation Component During Out-of-hospital Cardiac Arrest
}

\author{
Iraia Isasi ${ }^{1}$, Erik Alonso ${ }^{2}$, Unai Irusta ${ }^{1}$, Elisabete Aramendi ${ }^{1}$, Mohamud R. Daya $^{3}$ \\ ${ }^{1}$ Department of Communications Engineering, University of the Basque Country (UPV/EHU), \\ Bilbao, Spain \\ ${ }^{2}$ Department of Applied Mathematics, University of the Basque Country (UPV/EHU), Bilbao, Spain \\ ${ }^{3}$ Department of Emergency Medicine, Oregon Health \& Science University (OHSU), Portland, OR, \\ United States
}

\begin{abstract}
An automated pulse detector during out-of-hospital cardiac arrest (OHCA) is needed. The thoracic impedance (TI) recorded through defibrillation pads presents an impedance circulation component (ICC), hidden among other components, in the form of small fluctuations correlated with each effective heartbeat. This study presentes a method based on the stationary wavelet transform (SWT) to derive the ICC. A dataset with 456 5-s segments, 175 pulseless electrical activity (PEA) and 281 pulse-generating rhythm $(P R)$, with concurrent ECG and TI signals from 49 OHCA patients was used. The SWT was used to decompose the TI into 7 levels. The ICC was derived from soft denoised $d_{6}-d_{7}$ or $d_{7}$ detail coefficients for segments with heart rate $\geq 93$ bpm and $<93 \mathrm{bpm}$, respectively. Six features characterizing the amplitude and area of the ICC and its first derivative (dICC) were calculated. Their PEA/PR discrimination power was measured using the area under the curve $(A U C)$. These AUCs were compared with those obtained for the same features derived from the ICC/dICC extracted using an adaptive recursive least-squares (RLS) algorithm. The six features showed a mean (standard deviation) AUC of 0.91 (0.03) while RLSbased features yielded an AUC of 0.85 (0.07). Combining these ICC/dICC features with ECG features in a machine learning classifier might result in a robust pulse detector.
\end{abstract}

\section{Introduction}

Out-of-hospital cardiac arrest (OHCA) is one of the leading causes of death in the industralized world which in average accounts for the death of 83.7-95.9 per 100,000 person-years [1]. A rapid recognition of cardiac arrest is crucial for the survival of the patient as it allows for a prompt initation of cardiopulmonary resuscitation (CPR) and an early defibrillation which have been reported to im- prove survival $[2,3]$. Current resuscitation guidelines recommend assessing for the absence of 'normal breathing' as an indicator of the lack of circulation and therefore, cardiac arrest [4]. Unfortunately, this procedure has been reported unreliable [5] and introduces unnecessary CPR interruptions that are detrimental for patient's survival [6]. Therefore, the incorporation of a reliable pulse detector into defibrillators would contribute to a prompt and fast identification of the cardiac arrest, and would minimize CPR interruptions to confirm absence of circulation. Furthermore, it would allow for a rapid identification of the return of spontaneous circulation (ROSC), which would lead to an immediate initiation of post-resuscitation care.

In essence, pulse detection in cardiac arrest consists in discriminating between two types of organized rhythms: pulseless electrical activity (PEA) and pulse-generating rhythm (PR). Both rhythms present an apparently normal ECG with QRS complexes, PR presents both normal electrical and mechanical activity but PEA, unlike PR, shows an electromechanical dissociation, the heart loses its contractile function and therefore, there is no effective blood flow. The difference between PEA and PR is not always evident in the ECG. However, most of the current commercial defibrillators record the ECG and the thoracic impedance (TI) signals. The latter can help discriminate PEA and PR as it shows an impedance circulation component (ICC) consisting in small fluctuations $(<100 \mathrm{~m} \Omega)$ correlated with the QRS complexes for PR, but not for PEA. The ICC is hidden among other TI components.

The aim of this study was to develop a method to derive the ICC out of the TI signal that maximized its PEA/PR discrimination capability. Thus, in future work, waveform features extracted from this ICC might be combined with those obtained from the ECG and fed to a machine learning classifier to develop a robust and reliable pulse detector. 
PR
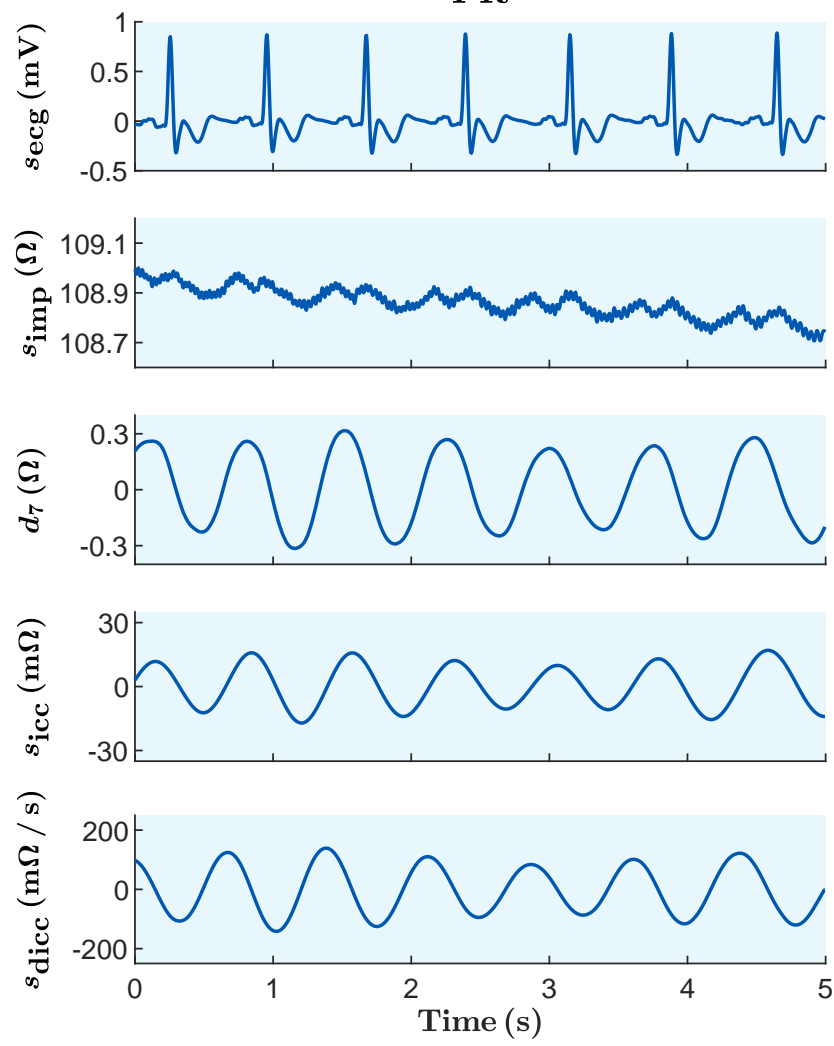

PEA
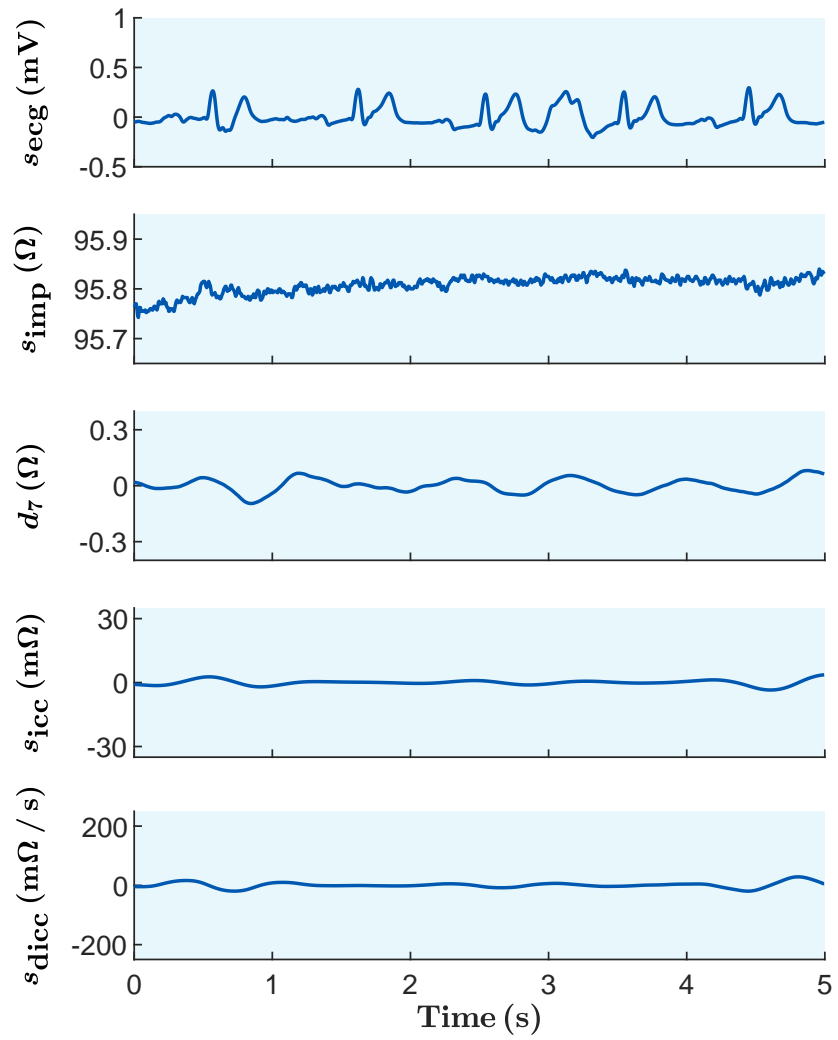

Figure 1. Examples of a PR (left) and PEA (right) segments where wavelet analysis of the TI signal was carried out. From top to bottom, the original ECG signal $\left(s_{\text {ecg }}\right)$, the original TI signal $\left(s_{\text {imp }}\right)$, a relevant subband of the TI $\left(d_{7}\right)$ for estimation of the ICC, the ICC $\left(s_{\text {icc }}\right)$ and its first derivative $\left(s_{\text {dicc }}\right)$.

\section{Materials and methods}

\subsection{Data materials}

The data used in the study were gathered from 49 OHCA patients treated by the Tualatin Valley Fire \& Rescue (Tigard, OR, USA) using the Philips HeartStart MRx (Philips Healthcare, MA, USA) monitor/defibrillator in 2012. Each episode contained concurrent ECG, TI and capnography signals resampled at a rate of $200 \mathrm{~Hz}$. Episodes were visually inspected to extract $5 \mathrm{~s}$ segments presenting QRS complexes and no artifacts due to chest compressions and ventilations. Those segments were labelled as PEA or PR by three expert reviewers that made a decision based on ROSC annotations made in the field and on the abrupt increases/decreases in the capnogram that were an indicator of ROSC and loss of ROSC, respectively. The dataset used in the study was composed of a total of 456 segments from which 175 corresponded to PEA and 281 to PR. Top panels of Figure 1 show the ECG and TI signals for a PR (left) and PEA (right).

\subsection{Extraction of the ICC}

The ICC was extracted from the TI signal, $s_{\text {imp }}(n)$, through the stationary wavelet transform (SWT) which consists in the application of a pair of low-pass, $h_{j-1}(n)$, and high-pass, $g_{j-1}(n)$, filters to produce, at the next level of decomposition, the approximation $\left(a_{j}\right)$ and detail $\left(d_{j}\right)$ coefficients:

$$
\begin{aligned}
& a_{j}(n)=h_{j-1}(n) * a_{j-1}(n) \\
& d_{j}(n)=g_{j-1}(n) * a_{j-1}(n)
\end{aligned}
$$

where $(*)$ stands for convolution, and $a_{0}(n)=s_{\text {imp }}(n)$. At stage $j$ the filters were the same as those of stage 0 but upsampled by a $2^{j}$ factor.

A Biorthogonal 4.4 mother wavelet was used to decompose $s_{\text {imp }}(n)$ into $J=7$ decomposition levels which produced detail coefficients $d_{1}-d_{7}$ and approximation coefficient $a_{7}$. Soft denoised detail coefficients $d_{6}-d_{7}$ $(0.78-3.13 \mathrm{~Hz}$ band) for segments with heart rate above $93 \mathrm{bpm}$ or simply $d_{7}(0.78-1.56 \mathrm{~Hz}$ band) for segments with heart rate below $93 \mathrm{bpm}$ were used to synthesize the 
ICC, $s_{\text {icc }}(n)$, by recursively evaluating

$$
a_{j-1}(n)=\frac{1}{2}\left(g_{j}^{\prime}(n) * a_{j}(n)+h_{j}^{\prime}(n) * d_{j}(n)\right)
$$

from $j=J, \ldots, 1$, where $g_{j}^{\prime}(n)$ and $h_{j}^{\prime}(n)$ are the synthesis filters. Bottom panels of Figure 1 show the most relevant signals obtained through the wavelet analysis of $s_{\text {imp }}$.

\subsection{Feature extraction}

A total of 6 features were computed from $s_{\text {icc }}(n)$ and its first derivative $s_{\text {dicc }}(n)$. The mean peak-to-trough amplitude (MPT/MPT*), the standard deviation (SD) of the peak-to-trough amplitude (SDPT/SDPT ${ }^{*}$ ) and mean area (MA/MA*) were computed from $s_{\text {icc }}(n) / s_{\text {dicc }}(n)$, respectively [7]. Features were computed following the description provided by original authors in the reference above and the Matlab implementation used in this study is available in https://github.com/ erik-alonso/PEA-PR-features.

\subsection{Evaluation of the disciminative power of ICC}

The PEA/PR discrimination power of the extracted features was measured in terms of area under the curve (AUC) of the Receiver Operating Characteristic Curve analysis and their sensitivity (SE, capacity to correctly detect PR), specificity (SP, capacity to correctly detect PEA) and balanced accuracy (BAC, mean value of SE and SP). These metrics were compared with those obtained for the same features derived from the $s_{\text {icc }}(n)$ and $s_{\text {dicc }}(n)$ extracted using the adaptive extraction based on a recursive least squares (RLS) algorithm proposed by Alonso et al. [8].

\section{Results and discussion}

Figure 2 shows the ROC curves for each of the 6 extracted features for our SWT-based method and for the RLS-based method. Table 2 describes in greater detail the discriminative power of the features extracted from our
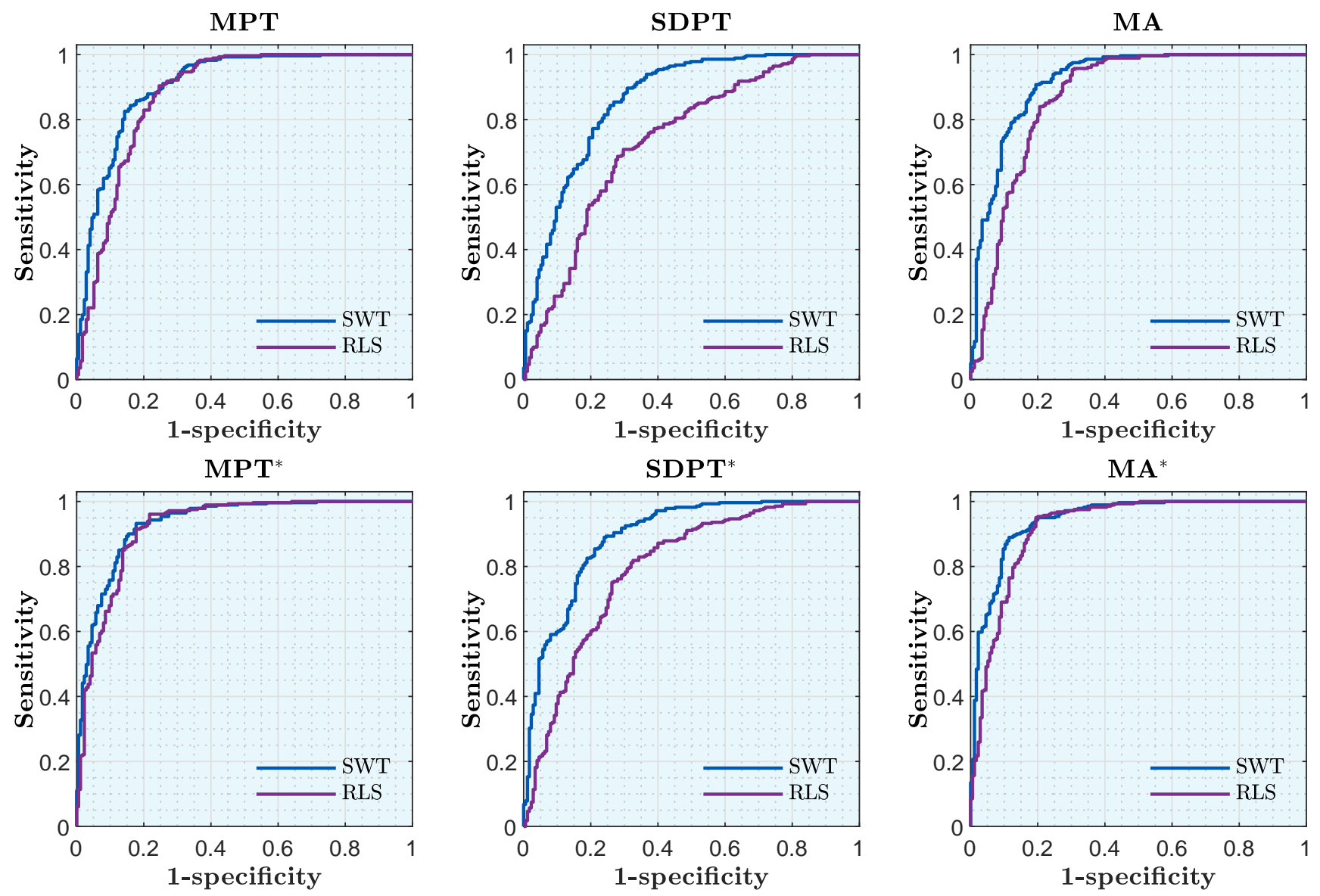

Figure 2. ROC curves for each of the extracted features for our method (SWT) and for the RLS-based method (RLS). From left to right, top panels correspond to features MPT, SDPT and MA extracted from $s_{\text {icc }}$ while bottom panels shows the ROC curves for features $\mathrm{MPT}^{*}, \mathrm{SDPT}^{*}$ and $\mathrm{MA}^{*}$ extracted from $s_{\mathrm{dicc}}$. 
Table 1. Discriminative power of the features extracted though the SWT-based method and via RLS-based method in terms of AUC, SE, SP and BAC.

\begin{tabular}{clcccc}
\hline Feature & & AUC & SE(\%) & SP(\%) & BAC(\%) \\
\hline \multirow{2}{*}{ MPT } & SWT & 0.91 & 84.3 & 84.0 & 84.2 \\
& RLS & 0.88 & 90.4 & 75.4 & 82.9 \\
\multirow{2}{*}{ SDPT } & SWT & 0.86 & 89.7 & 69.1 & 79.4 \\
& RLS & 0.74 & 70.8 & 70.3 & 70.6 \\
MA & SWT & 0.92 & 90.7 & 80.6 & 85.7 \\
& RLS & 0.87 & 95.4 & 69.7 & 82.5 \\
MPT $^{*}$ & SWT & 0.93 & 93.2 & 82.3 & 87.8 \\
& RLS & 0.92 & 96.1 & 78.3 & 87.2 \\
SDPT $^{*}$ & SWT & 0.89 & 89.0 & 76.0 & 82.5 \\
& RLS & 0.80 & 81.9 & 67.4 & 74.6 \\
MA $^{*}$ & SWT & 0.94 & 89.0 & 88.6 & 88.8 \\
& RLS & 0.92 & 95.0 & 80.6 & 87.8 \\
\hline
\end{tabular}

method and from the RLS-based method. The mean (SD) AUC presented by the set of features was $0.91(0.03)$ and 0.85 (0.07) for the SWT-based and the RLS-based methods, respectively. Features extracted by our method also showed greater capability to discriminate between PEA and PR compared to those extracted by the RLS-based method. In average, SWT-based features presented a BAC 4-points above that obtained by RLS-based features, a mean(SD) BAC of 84.7 (3.5) and 80.9(6.9), respectively. Every feature extracted through our method presented greater discriminative power than that obtained by its analogous using the RLS method.

\section{Conclusions}

This study proposes a new method based on the SWT to extract the ICC from the TI signal. The goodness of the method was evaluated in terms of the PEA/PR discrimination capability of 6 well-known features extracted from the ICC and its first derivative. These features showed great discriminative power that was above that obtained by features extracted using an adaptive RLS solution proposed in [8]. Therefore, future work should take advantage of these promising results and combine features from the SWT-based ICC and ECG features to improve the pulse detection algorithms presented in the literature.

\section{Acknowledgments}

This work was supported partially by the Spanish Ministry of Science, Innovation and Universities through Grant RTI2018-101475-BI00, jointly with the Fondo Europeo de Desarrollo Regional (FEDER), and in part by the Basque
Government through Grant IT-1229-19.

\section{References}

[1] Berdowski J, Berg RA, Tijssen JGP, Koster RW. Global incidences of out-of-hospital cardiac arrest and survival rates: Systematic review of 67 prospective studies. Resuscitation 2010;81(11):1479-1487.

[2] Wissenberg M, Lippert FK, Folke F, Weeke P, Hansen CM, Christensen EF, Jans H, Hansen PA, Lang-Jensen T, Olesen JB, Lindhardsen J, Fosbol EL, Nielsen SL, Gislason GH, Kober L, Torp-Pedersen C. Association of National Initiatives to Improve Cardiac Arrest Management With Rates of Bystander Intervention and Patient Survival After Out-of-Hospital Cardiac Arrest. JAMA October 2013; 310(13):1377-1384.

[3] Blom MT, Beesems SG, Homma PCM, Zijlstra JA, Hulleman M, van Hoeijen DA, Bardai A, Tijssen JGP, Tan HL, Koster RW. Improved survival after out-of-hospital cardiac arrest and use of automated external defibrillators. Circulation November 2014;130(21):1868-1875.

[4] Perkins GD, Handley AJ, Koster RW, Castrén M, Smyth MA, Olasveengen T, Monsieurs KG, Raffay V, Gräsner JT, Wenzel V, Ristagno G, Soar J, Adult basic life support and automated external defibrillation section Collaborators. European Resuscitation Council Guidelines for Resuscitation 2015: Section 2. Adult basic life support and automated external defibrillation. Resuscitation October 2015;95:81-99.

[5] Ruppert M, Reith MW, Widmann JH, Lackner CK, Kerkmann R, Schweiberer L, Peter K. Checking for breathing: evaluation of the diagnostic capability of emergency medical services personnel, physicians, medical students, and medical laypersons. Annals of Emergency Medicine December 1999;34(6):720-729.

[6] Vaillancourt C, Everson-Stewart S, Christenson J, Andrusiek D, Powell J, Nichol G, Cheskes S, Aufderheide TP, Berg R, Stiell IG, Resuscitation Outcomes Consortium Investigators. The impact of increased chest compression fraction on return of spontaneous circulation for out-of-hospital cardiac arrest patients not in ventricular fibrillation. Resuscitation December 2011;82(12):1501-1507.

[7] Ruiz J, Alonso E, Aramendi E, Kramer-Johansen J, Eftestøl T, Ayala U, González-Otero D. Reliable extraction of the circulation component in the thoracic impedance measured by defibrillation pads. Resuscitation October 2013; 84(10):1345-1352.

[8] Alonso E, Aramendi E, Daya M, Irusta U, Chicote B, Russell JK, Tereshchenko LG. Circulation detection using the electrocardiogram and the thoracic impedance acquired by defibrillation pads. Resuscitation February 2016;99:56-62.

Address for correspondence:

Iraia Isasi

Plaza Ingeniero Torres Quevedo 1, 48013 Bilbao, Spain

iraia.isasi@ehu.eus 